\title{
Design and Stability Analysis of Uncertain Networked Predictive Control Systems with Multiple Forward Channels
}

\author{
Hongbo Song \\ College of Information, Zhejiang University of Finance and Economics, Hangzhou 310018, China \\ Correspondence should be addressed to Hongbo Song; di7ganshb@163.com
}

Received 2 January 2014; Accepted 24 February 2014; Published 2 April 2014

Academic Editor: Huaicheng Yan

Copyright (C) 2014 Hongbo Song. This is an open access article distributed under the Creative Commons Attribution License, which permits unrestricted use, distribution, and reproduction in any medium, provided the original work is properly cited.

\begin{abstract}
This paper is concerned with the design and stability of networked predictive control for uncertain systems with multiple forward channels. The delays and packet dropouts are distributed such that the classic networked predictive control (NPC) needs modifications to be implemented. An improved control signal selection scheme with distributed prediction length is proposed to increase the prediction accuracy and hence achieve better control performance. Moreover, stability analysis results are obtained for both constant and random cases. Interestingly, it is shown that the stability of the closed-loop NPC system is not related to the distributed delays when they are constant and the system model is accurate. Finally, a two-axis milling machine example is given to illustrate the effectiveness of the proposed method.
\end{abstract}

\section{Introduction}

As modern control systems become more and more complex, traditional point-to-point control architecture is no longer suitable under certain circumstances. Meanwhile, the technology of computer network improved significantly in the past decades and a new networked architecture emerges and attracts increasing attention. This kind of control systems are called networked control systems (NCSs), in which the control loops are closed via a network, for example, Field bus, Ethernet, and Internet [1-3]. Major advantages of NCSs include reduced cost, easy installation and maintenance, and high efficiency. However, the insertion of a network into the control loops introduces some challenging problems for NCSs such as network-induced delay, packet dropout, and quantization [4-7]. Moreover, beside the control problem for NCSs, state estimation or filtering for NCSs also attracts much attention [8-10]. Thus, NCSs have become a hot research area in the control and signal processing communities.

Among these problems, network-induced delay and packet dropout are two major issues that degrade the system performance or even cause instability [1]. Up to date, both network-induced delay and packet dropout issues have received extensive research attention and fruitful results were obtained. To name a few, time delay method $[11,12]$, stochastic system method [13], switched system method [14], and robust control method [15] were proposed to deal with the delay. Switched system method [16] and stochastic system method [17] were presented to handle the packet dropout. These methods commonly model the delay or/and packet dropout into the closed-loop NCS and the corresponding controller were then designed based on conditions that make the closedloop NCS stable. Most of the conditions are only sufficient and thus the design is conservative. Moreover, essentially, they all passively compensate for the negative effects of the delay and packet dropout after accepting them. Alternatively, a novel actively compensating method called networked predictive control (NPC) was presented in $[18,19]$. In NPC, future control sequences are generated and transmitted in a packet through the network. At the plant side, appropriate control signals from the sequences are selected to control the plant based on delay measurements.

With accurate system model and delay measurements, NPC was shown to have the ability to achieve desired control performance and the stability of the closed-loop NPC system is not related to the delay and packet dropout in the constant case [20]. In [21], an event driven NPC was presented to avoid the practical problem that delay measurements are inaccurate. In [22], a switched controller structure was proposed and the controllers were designed 
according to the delay based on switched system method. For more research results on NPC, see [23-28] and the references therein. However, two issues of NPC are not fully considered in the existing literature, which are the model uncertainty and multiple communication channels. The NPC for uncertain system with multiple distributed delays and packet dropouts in the feedback channels was studied in [29]. In [30], stability analysis was carried out for NPC systems with model uncertainty. In [31], a data reconstruction method was presented for NPC system with distributed delays in the feedback channels.

For a practical system, it is very difficult to obtain an accurate model. A nonlinear system is always linearized to be simplified. Thus, model uncertainty is an important issue to be investigated. On the other hand, when the plant to be controlled is spatially distributed, the corresponding NCSs have to be multiple communication channels. Obviously, for a NPC system with model uncertainty, larger input delay results in longer prediction length and thus leads to larger prediction error. Hence, the control performance becomes worse. In the case of multiple communication channels, delays and packet dropouts are distributed, which means that delays and packet dropout process may be different for each channel, while such case was not taken into account in classic NPC such as [18-20]. Introducing the queuing method in [32] can make the input delays the same for each channel, which makes the classic NPC applicable for the case without modification. However, input delays are actually enlarged in this way and the control performance will hence be degraded. Thus, this paper presents a modified control signal selection scheme such that the control signals which are predicted with distributed prediction length can be applied to control the plant. Such treatment essentially uses the most recent data and hence reduces the prediction length and improves the system performance. Only multiple forward channels are considered since the compensation scheme in the forward channels is different from the one in the feedback channels.

\section{Design of NPC for Systems with Multiple Forward Channels}

2.1. Structure of the NPC System. The structure of NPC systems with multiple forward channels is shown in Figure 1. The feedback channels are assumed to be ideal such that the plant outputs are transmitted to the predictive controller (PC) side without any delays or packet dropouts. The task of PC is to generate future control prediction sequences and transmit them to the control signal selector (CSS). The sequences are subjected to distributed delays and packet dropouts effects since the system has multiple forward channels. CSS receives the distributed prediction sequences and select proper control signal from them according to the measured input delays. The selected control signal is then used to control the plant.

The control performance of NPC systems highly depends on the prediction accuracy, which is related to both the discrepancy between the adopted model and real system dynamic and the prediction length. On the other hand, larger input delay results in longer prediction length. Based on the
NPC in $[19,30]$, it is known that larger modeling error and larger delay lead to larger prediction errors and hence degrade the control performance. Thus, intuitively, it can be inferred that two ways to improve the control performance of NPC systems are to reduce the modeling error and to make the prediction length shorter.

For the NPC systems with multiple forward channels, the delays and packet dropouts process are distributed. If we use the classic NPC, which assumes single communication channel, the input delays of all the channels should be the same and equal to a value which is the largest one of the distributed input delays. This can be done by the queuing method. However, with this treatment it can be seen that the prediction length is enlarged for the channels except the one with largest input delays. Based on the thought that shorter prediction length leads to better prediction accuracy, another way is to use an improved CSS with distributed prediction length, which makes the prediction length shorter than using classic NPC. This is the main idea of the paper.

The plant is represented by the following uncertain linear discrete-time state space system model:

$$
\begin{aligned}
x(k+1) & =(A+\Delta A) x(k)+(B+\Delta B) u(k), \\
y(k) & =C x(k),
\end{aligned}
$$

where $x \in R^{n}, u \in R^{m}$, and $y \in R^{p}$ are the state, input, and output vectors of the plant, respectively, and $A, B$, and $C$ are the nominal system matrices with compatible dimensions. $\Delta A$ and $\Delta B$ represent the system uncertainties and it is assumed that $\Delta A$ and $\Delta B$ satisfy the following structure:

$$
\left[\begin{array}{ll}
\Delta A & \Delta B
\end{array}\right]=\operatorname{EF}(k)\left[\begin{array}{ll}
G_{1} & G_{2}
\end{array}\right],
$$

where $F(k)^{T} F(k) \leq I$, and $E, G_{1}$ and $G_{2}$ are matrices with compatible dimensions.

The prediction sequences are designed based on the following standard state observer:

$$
\begin{aligned}
\widehat{x} & (k+1 \mid k) \\
& =A \widehat{x}(k \mid k-1)+B u(k)+L(y(k)-C \widehat{x}(k \mid k-1)),
\end{aligned}
$$

where $\widehat{x}(k \mid k-1)$ is the state of the observer, and $L$ is the observer gain matrix which can be designed by standard methods such as pole placement. The state feedback controller is as follows:

$$
u(k)=-K \widehat{x}(k \mid k-1),
$$

where $K$ is the controller gain matrix which can be designed by Lyapunov method and so on.

2.2. Assumptions and Notations. Before proceeding further, we need to introduce some reasonable assumptions and some notations.

(1) Without loss of generality, it is assumed that there are $m$ forward channels in the system and they are denoted by channel 1 to channel $m$, respectively. $u_{j}$ are transmitted through channel $j, j \in M=$ $\{1,2, \ldots, m\}$, respectively. 


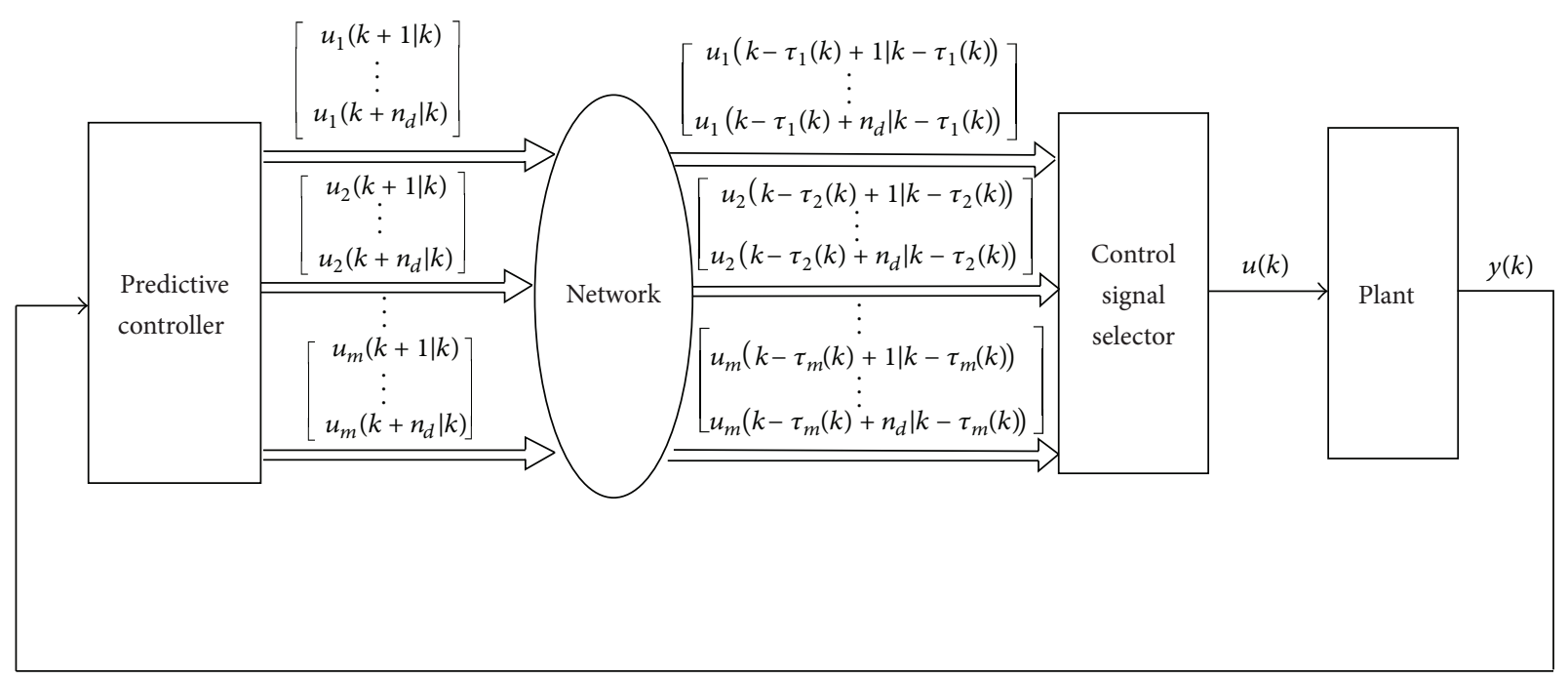

FIGURE 1: Structure of NPC systems with multiple forward channels.

(2) The distributed delays are bounded. The upper bound for the delays is denoted by $n_{d}$ and the lower bound is assumed to be 1 step without loss of generality.

(3) The number of consecutive packet dropouts for channels 1 to $m$ is bounded and the upper bound is denoted by $n_{p}$. The lower bound is assumed to be 0 steps, which means no packet dropout.

(4) The transmitted packets are time-stamped and the clocks of the receiving sides and the transmitting sides are synchronized such that the input delays can be calculated.

At any time instant, it is probable that more than one packet are received or no packet is received because of packet dropouts or packet disorder. In both cases, the data in the most recent packet will be used. The input delays then can always be calculated by the time stamp.

Since the case random delays and packet dropouts are considered, the input delay for channel $i$ at instant $k$ is denoted by $\tau_{i}(k)$ and it is clear that the lower and upper bound for the input delays are 1 and $n_{f}$, respectively, where $n_{f}=n_{d}+$ $n_{p}$. It can be seen that input delays may be of the same value for certain channels. That is to say, $\tau_{i}(k)=\tau_{j}(k), i, j \in M$. Thus, we denote the number of different values for the input delays as $d(k)$. The corresponding values for the input delays are denoted by $\rho_{1}(k), \rho_{2}(k), \ldots, \rho_{d(k)}(k)$, respectively, where $\rho_{i}(k)<\rho_{j}(k)$ for any $i<j, i, j \in M$. The set of the indexes of the channels in which $\rho_{i}(k)=\tau_{j}(k)$ is denoted by $Q_{i}(k)$. Introduce selection matrices $\Pi_{j}, \forall j \in M$, which is defined by

$$
\Pi_{j}=\operatorname{diag}\{\delta(j-1), \delta(j-2), \ldots, \delta(j-m)\},
$$

where $\operatorname{diag}\{\bullet\}$ represents a diagonal matrix and $\delta(j)=$ $\begin{cases}1, & j=0 \\ 0, & j \neq 0 .\end{cases}$

An example is given to make these notations clear. Assume that the system has 3 forward channels, which means $m=3$. At instant $k$, it is measured that $\tau_{1}(k)=3$ and $\tau_{2}(k)=\tau_{3}(k)=2$. Then, it can be seen that there are two different values for the distributed delays; that is, $d(k)=2$ and $\rho_{1}(k)=2, \rho_{2}(k)=3$. Furthermore, we have $Q_{1}(k)=\{2,3\}$ and $Q_{2}(k)=\{1\}$, respectively.

For the constant delays case, the notations can be simply used by removing the index $k$ of the ones for the random delays and packet dropouts case.

2.3. Predictive Controller. At the $\mathrm{PC}$ side, the state of the observer $\widehat{x}(k+1 \mid k)$ is obtained at instant $k$ according to (3). Since future system outputs $y(k+i)$ and $i=1,2, \ldots, n_{f}-1$ are not available at instant $k$, the prediction sequences are generated as follows:

$$
\begin{gathered}
\widehat{x}(k+2 \mid k)=A \hat{x}(k+1 \mid k)+B u(k+1 \mid k) \\
\widehat{x}(k+3 \mid k)=A \hat{x}(k+2 \mid k)+B u(k+2 \mid k) \\
\vdots \\
\widehat{x}\left(k+n_{f} \mid k\right)=A \widehat{x}\left(k+n_{f}-1 \mid k\right)+B u\left(k+n_{f}-1 \mid k\right) .
\end{gathered}
$$

The prediction is up to $n_{f}$ steps to meet the worst case of delays and packet dropouts, that is, $n_{d}$ steps delays and $n_{p}$ consecutive packet dropouts. For the prediction procedure (6), it can be seen that the prediction accuracy at each instant depends on two factors. The first is how accurate $(A, B, C)$ represents the system dynamic. The second is how close $u(k+$ $i \mid k)$ to $u(k+i), \forall i \in N=\left\{1,2, \ldots, n_{f}\right\}$. It is interesting to see that $u(k+i \mid k)$ are fundamentally different for the random and constant cases.

For the constant delays case, even though the future control input $u(k+i)$ is not applied, it can be inferred what control signal will be applied at instant $k+i$ and the control signal is already available at the PC side. That is to say, PC can use $u(k+i)$ instead of $u(k+i \mid k)$ in (6). For the random case, $\mathrm{PC}$ cannot determine what control signal will be used at 
instant $k+i, \forall i \in N$. Thus, $u(k+i \mid k)$ can be approximated by (4) such that

$$
u(k+i \mid k)=-K \hat{x}(k+i \mid k) .
$$

This will also lead to different techniques used for the stability analysis of random and constant cases.

In classic NPC, the future control sequence is transmitted in a packet to the CSS as follows:

$$
\left[\begin{array}{c}
u(k+1 \mid k) \\
u(k+2 \mid k) \\
\vdots \\
u\left(k+n_{f} \mid k\right)
\end{array}\right]
$$

However, this paper considers the multiple forward channels case and thus the packet that contains $u(k+i \mid k), \forall i \in N$, is transmitted separately via $m$ channels. The packets transmitted through channel $i$ contains the following information, respectively. Consider

$$
\begin{aligned}
& {\left[\begin{array}{c}
u_{1}(k+1 \mid k) \\
u_{1}(k+2 \mid k) \\
\vdots \\
u_{1}\left(k+n_{f} \mid k\right)
\end{array}\right],} \\
& {\left[\begin{array}{c}
u_{2}(k+1 \mid k) \\
u_{2}(k+2 \mid k) \\
\vdots \\
u_{2}\left(k+n_{f} \mid k\right)
\end{array}\right] \ldots,\left[\begin{array}{c}
u_{m}(k+1 k) \\
u_{m}(k+2 k) \\
\vdots \\
u_{m}\left(k+n_{f} k\right)
\end{array}\right] .}
\end{aligned}
$$

2.4. Control Signal Selector. Subjected to the distributed delays and packet dropouts effects, the packets are received by the CSS in a distributed delayed manner. Specifically, the following data are received by CSS at instant $k$ :

$$
\left[\begin{array}{c}
u_{i}\left(k-\tau_{i}(k)+1 \mid k-\tau_{i}(k)\right) \\
u_{i}\left(k-\tau_{i}(k)+2 \mid k-\tau_{i}(k)\right) \\
\vdots \\
u_{i}\left(k-\tau_{i}(k)+n_{f} \mid k-\tau_{i}(k)\right)
\end{array}\right], \quad \forall i \in M .
$$

As mentioned, classic NPC with using queuing method can be used and in this case the selected control signal is

$$
u(k)=u(k \mid k-\bar{\tau}(k))
$$

where $\bar{\tau}(k)=\max _{i \in M} \tau_{i}(k)$.

Alternatively, a modified CSS scheme is presented here. We can see that for channel $i, \forall i \in M$, the predicted control signal with minimal prediction length is

$$
u_{i}(k)=u_{i}\left(k \mid k-\tau_{i}(k)\right) .
$$

Then, it follows by using the notations in the second subsection of this section that

$$
u_{i}(k)=\sum_{j \in Q_{i}(k)} \Pi_{j} u\left(k \mid k-\rho_{i}(k)\right) .
$$

It can be seen that the modified CSS selects control signal with distributed prediction length. Then, we can see that the following control signal to control the plant:

$$
u(k)=-\sum_{i=1}^{d(k)} \sum_{j \in Q_{i}(k)} \Pi_{j} K \hat{x}\left(k \mid k-\rho_{i}(k)\right) .
$$

As a special case, if $\rho_{i}(k)=\rho_{j}(k), \forall i \in M, j \in M /\{i\}$, which means that the length of the delays are all the same for each channel, then it can be seen that the control input (14) equals the control input (11).

\section{Stability Analysis of the Closed-Loop NPC System}

The last section designed a modified NPC strategy to control uncertain systems with multiple forward channels. Since stability is crucial for a control system, the stability of the closed-loop NPC system is studied in this section. Two cases are considered, which are the random distributed delays and packet dropouts case and the constant distributed delays case. As mentioned in the above section, the analysis techniques are different for the two cases.

3.1. Model of the Closed-Loop NPC System: Random Case. By (6) and (7), it follows that

$$
\widehat{x}(k+i \mid k)=(A-B K)^{i-1} \widehat{x}(k+1 \mid k) .
$$

Then, by (14) and (15) we have

$$
\begin{aligned}
u(k)= & -\sum_{i=1}^{d(k)} \sum_{j \in \mathrm{Q}_{i}(k)} \Pi_{j} K(A-B K)^{\rho_{i}(k)-1} \\
& \cdot \widehat{x}\left(k-\rho_{i}(k)+1 \mid k-\rho_{i}(k)\right) .
\end{aligned}
$$

Define an augmented vector to be

$$
\xi(k)=\left[\begin{array}{c}
x(k) \\
x(k \mid k-1) \\
x(k-1 \mid k-2) \\
\vdots \\
x\left(k-n_{f}+1 \mid k-n_{f}\right)
\end{array}\right],
$$

then the closed-loop NPC systems with random distributed delays and packet dropouts can be obtained by (1), (3), and (16) as follows:

$$
\xi(k+1)=(\Omega(k)+\Delta \Omega(k)) \xi(k),
$$

where

$$
\begin{gathered}
\Omega(k)=\left[\begin{array}{cc}
\Omega_{11} & B \Omega_{12}(k) \\
\Omega_{21} & \Omega_{22}
\end{array}\right], \\
\Omega_{11}=\left[\begin{array}{c}
A \\
L C
\end{array}\right], \\
\Omega_{21}=\left[\begin{array}{l}
0_{\left(n_{f}-2\right) n \times n}
\end{array}\right], \\
\Omega_{22}=\left[I_{\left(n_{f}-2\right) n \times\left(n_{f}-2\right) n} 0_{\left(n_{f}-2\right) n \times n}\right],
\end{gathered}
$$




$$
\begin{aligned}
& \Omega_{12}(k)=\Omega_{121}+\Omega_{122}(k), \\
& \Omega_{121}=\left[\begin{array}{ccccc}
0_{n \times n} & 0_{n \times n} & 0_{n \times n} & \ldots & 0_{n \times n} \\
A-L C & 0_{n \times n} & 0_{n \times n} & \ldots & 0_{n \times n}
\end{array}\right], \\
& \Omega_{122}(k) \\
& =\left[\begin{array}{lllll}
\Omega_{1221}(k) & \Omega_{1222}(k) & \ldots & \Omega_{122 d(k)}(k) & 0_{n \times\left(n_{f}-d(k)\right) n} \\
\Omega_{1221}(k) & \Omega_{1222}(k) & \ldots & \Omega_{122 d(k)}(k) & 0_{n \times\left(n_{f}-d(k)\right) n}
\end{array}\right], \\
& \Omega_{1221}(k)=\left[\begin{array}{ll}
0_{\left(\rho_{1}(k)-1\right) n \times\left(\rho_{1}(k)-1\right) n} & \Omega_{12212}(k)
\end{array}\right], \\
& \Omega_{1222}(k)=\left[\begin{array}{ll}
0_{\left(\rho_{2}(k)-1\right) n \times\left(\rho_{2}(k)-1\right) n} & \Omega_{12222}(k)
\end{array}\right],
\end{aligned}
$$

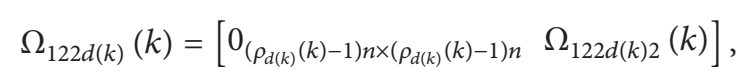

$$
\begin{aligned}
& \Omega_{12212}(k)=\sum_{j \in \mathrm{Q}_{1}(k)} \Pi_{j} K(A-B K)^{\rho_{1}(k)-1}, \\
& \Omega_{12222}(k)=\sum_{j \in \mathrm{Q}_{2}(k)} \Pi_{j} K(A-B K)^{\rho_{2}(k)-1}, \\
& \Omega_{122 d(k) 2}(k)=\sum_{j \in Q_{d(k)}(k)} \Pi_{j} K(A-B K)^{\rho_{d(k)}(k)-1}, \\
& \Delta \Omega(k)=\left[\begin{array}{cc}
\Delta A & \Delta B \Delta \Omega_{12}(k) \\
0_{n_{f} n \times n} & 0_{n_{f} n \times n_{f} n}
\end{array}\right], \\
& \Delta \Omega_{12}=\left[\begin{array}{cc}
I_{n \times n} & 0_{n \times n} \\
0_{n \times n} & 0_{n \times n}
\end{array}\right] * \Omega_{122}(k) \text {. }
\end{aligned}
$$

Note that the dynamics of the closed-loop NPC system (18) is related to the input delays vector $\vec{\rho}(d(k), k)=$ $\left[\begin{array}{llll}\rho_{1}(k) & \rho_{2}(k) & \cdots & \rho_{d(k)}(k)\end{array}\right]$; denote the set of all the possible values of $\vec{\rho}(d(k), k)$ as $V$. When $\vec{\rho}(d(k), k)$ takes a value in $V$, system (18) resides in the corresponding subsystem, which means that system (18) is a switched system. For simplicity of notation, introduce a one-to-one mapping that maps the set of $\vec{\rho}(d(k), k)$ to a set with numbers. For example, $[1,1, \ldots, 1]$ is mapped to $1,[1,1, \ldots, 2]$ is mapped to 2 , and so on. Denote the set of the numbers by $C$. Moreover, the system has model uncertainties. Thus, system (18) can be transformed into the following uncertain switched system:

$$
\xi(k+1)=\left(\Phi_{s}+\Delta \Phi_{s}\right) \xi(k), \quad s \in C,
$$

where

$$
\begin{aligned}
\Phi_{s} & =\left[\begin{array}{ll}
\Omega_{11} & \Phi_{12 s} \\
\Omega_{21} & \Omega_{22}
\end{array}\right], \\
\Delta \Phi_{s} & =\left[\begin{array}{cc}
\Delta A & \Delta \Phi_{12 s} \\
0_{n_{f} n \times n} & 0_{n_{f} n \times n}
\end{array}\right],
\end{aligned}
$$

and $\Phi_{12 s}$ and $\Delta \Phi_{12 s}$ are $B \Omega_{12}(k)$ and $\Delta B \Delta \Omega_{12}(k)$ with $\vec{\rho}(d(k), k)$ is mapped by $s, \forall s \in C$.
3.2. Stability Results: Random Case. The closed-loop NPC system with random distributed delays and packet dropouts in the multiple forward channels is modeled as the switched uncertain system (20). A sufficient condition for the stability of the closed-loop NPC system (20) is presented in this subsection. A lemma is first given as follows.

Lemma 1. For given appropriate matrices $\Upsilon_{1}, \Upsilon_{2}$, and $\Upsilon_{3}$, with $\Upsilon_{1}^{T}=\Upsilon_{1}$,

$$
\Upsilon_{1}+\Upsilon_{2} F(k) \Upsilon_{3}+\Upsilon_{3}^{T} F^{T}(k) \Upsilon_{2}^{T}<0
$$

holds for all $F^{T}(k) F(k) \leq I$ if and only if there exists a scalar $\beta>0$ such that

$$
\Upsilon_{1}+\beta \Upsilon_{2} \Upsilon_{2}^{T}+\beta^{-1} \Upsilon_{3}^{T} \Upsilon_{3}<0
$$

Theorem 2. For the NPC system (20) with random distributed delays and packet dropouts, given all possible values of $\vec{\rho}(d(k), k)$, and controller gain matrix $K$ and observer gain matrix $L$, if there exists a matrix $X$ and a scalar $\beta>0$ such that the following matrix inequalities

$$
\left[\begin{array}{ccc}
-X & X \Phi_{s}^{T} & X \bar{G}_{s}^{T} \\
\Phi_{s} X & -X+\beta \overline{E E}^{T} & 0 \\
\bar{G}_{s} X & 0 & -\beta I
\end{array}\right]<0, \quad \forall s \in C
$$

hold, then the closed-loop NPC system (20) is stable.

Proof. It can be obtained by (2) that $\Delta \Omega_{s}$ has the structure $\Delta \Phi_{s}=\bar{E} F(k) \bar{G}_{s}$, where

$$
\bar{E}=\left[\begin{array}{c}
E \\
0_{n_{f} n \times n}
\end{array}\right], \quad \bar{G}_{s}=\left[\begin{array}{ll}
G_{1} & G_{2} \\
\Phi_{12 s}
\end{array}\right] .
$$

By the stability result of switched system with arbitrary switching in [10], if there exists a matrix $P>0$ such that the following

$$
\left(\Phi_{s}+\Delta \Phi_{s}\right)^{T} P\left(\Phi_{s}+\Delta \Phi_{s}\right)-P<0
$$

holds, then the closed-loop NPC system (20) is stable. Then, by Schur complement and some matrix operations, we have

$$
\left[\begin{array}{cc}
-P & \Phi_{s}^{T} \\
\Phi_{s} & -P^{-1}
\end{array}\right]+\left[\begin{array}{l}
0 \\
\bar{E}
\end{array}\right] F\left[\begin{array}{ll}
\bar{G}_{s} & 0
\end{array}\right]+\left[\begin{array}{c}
\bar{G}_{s}^{T} \\
0
\end{array}\right] F^{T}\left[\begin{array}{ll}
0 & \bar{E}^{T}
\end{array}\right]<0 .
$$

Then, by Lemma 1 it can be obtained that

$$
\left[\begin{array}{ccc}
-P & \Phi_{s}^{T} & \bar{G}_{s}^{T} \\
\Phi_{s} & -P^{-1}+\beta \overline{E E}^{T} & 0 \\
\bar{G}_{s} & 0 & -\beta I
\end{array}\right]<0 .
$$

Pre- and postmultiplying of (28) by $\left[\begin{array}{lll}P^{-1} & I & I\end{array}\right]$ and letting $X=P^{-1}$ lead to (24). The proof is completed.

It should be pointed out that (24) is linear in matrix $X$ and scalar $\beta$ and thus can be conveniently solved by LMI toolbox in Matlab for example. This means that the stability of the closed-loop NPC system with random distributed delays and packet dropouts can be readily checked. However, a common Lyapunov function is used in the proof, which leads to some conservatism. One possible way to reduce conservatism is to use multiple Lyapunov function method. 
3.3. Stability Results: Constant Case. In this section, the constant case is considered. Different from the random case, a necessary and sufficient condition is obtained. Define the state error as

$$
e(k)=x(k)-\widehat{x}(k \mid k-1) .
$$

Subtracting (3) from (1) leads to the following state error equation:

$$
e(k+1)=(A-L C) e(k)+\Delta A x(k)+\Delta B u(k) .
$$

For an integer $\tau \geq 2$, it can be seen by (6) that the following two equalities hold:

$$
\begin{aligned}
\hat{x}(k \mid k-\tau)= & A^{\tau-1} \hat{x}(k-\tau+1 \mid k-\tau) \\
& +\sum_{i=1}^{\tau-1} A^{i} B u(k-i), \\
\widehat{x}(k \mid k-\tau+1)= & A^{\tau-2} \widehat{x}(k-\tau+2 \mid k-\tau+1) \\
& +\sum_{i=1}^{\tau-2} A^{i} B u(k-i) .
\end{aligned}
$$

By shifting (3) backward for $\tau$ steps, it can be obtained that

$$
\begin{aligned}
\widehat{x}(k-\tau+2 \mid k-\tau+1) \\
=A \widehat{x}(k-\tau+1 \mid k-\tau) \\
\quad+B u(k-\tau+1)+L C e(k-\tau+1) .
\end{aligned}
$$

Then, by (33) and subtracting (31) from (32) we have the following:

$$
\widehat{x}(k \mid k-\tau+1)-\widehat{x}(k \mid k-\tau)=A^{\tau-2} L C e(k-\tau+1) .
$$

Applying (34) recursively results in the following equation that holds for any integer $\tau \geq 2$ :

$$
x(k \mid k-1)=x(k \mid k-\tau)+\sum_{i=1}^{\tau-1} A^{i-1} L C e(k-i) .
$$

We now have by (14) and (35)

$$
\begin{aligned}
u(k)= & -K \hat{x}(k \mid k-1) \\
& +\sum_{i=1}^{d} \sum_{j \in Q_{i}} \Pi_{j} K \sum_{l=1}^{\rho_{i}-1} A^{l-1} L C e(k-l) .
\end{aligned}
$$

For convenience of the representation of the closed-loop NPC system, $u(k)$ is transformed as follows:

$$
\begin{aligned}
u(k)= & -K \widehat{x}(k \mid k-1)+\sum_{l=1}^{\rho_{1}-1} A^{l-1} L C e(k-l) \\
& +\sum_{i=1}^{d-1} \sum_{j \in W_{i}} \Pi_{j} K \sum_{l=\rho_{i}}^{\rho_{i+1}-1} A^{l-1} L C e(k-l),
\end{aligned}
$$

where $W_{i}=Q_{i+1} \cup Q_{i+2} \cup \cdots \cup Q_{d}, i=1,2, \ldots, d$. Substituting $u(k)$ in (1) and (30), respectively, by (26) yields

$$
\begin{aligned}
x(k+ & 1) \\
= & (A-B K+\Delta A-\Delta B K) x(k)+(B+\Delta B K) e(k) \\
& +\sum_{l=1}^{\rho_{1}-1}(B+\Delta B) K A^{l-1} L C e(k-l) \\
& +\sum_{i=1}^{d-1} \sum_{j \in W_{i}} B \Pi_{j} K \sum_{l=\rho_{i}}^{\rho_{i+1}-1} A^{l-1} L C e(k-l) \\
& +\sum_{i=1}^{d-1} \sum_{j \in W_{i}} \Delta B \Pi_{j} K \sum_{l=\rho_{i}}^{\rho_{i+1}-1} A^{l-1} L C e(k-l) \\
e(k+ & 1) \\
= & (A-L C) e(k)+(\Delta A-\Delta B K) x(k)-\Delta B K e(k) \\
& +\sum_{l=1}^{\rho_{1}-1} \Delta B K A^{l-1} L C e(k-l) \\
& +\sum_{i=1}^{d-1} \sum_{j \in W_{i}} \Delta B \Pi_{j} K \sum_{l=\rho_{i}}^{\rho_{i+1}-1} A^{l-1} L C e(k-l) .
\end{aligned}
$$

Define an augmented vector as

$$
\begin{aligned}
& \eta(k)=\left[\begin{array}{llllll}
x^{T}(k) & e^{T}(k) & \eta_{1}^{T}(k) & \eta_{2}^{T}(k) & \cdots & \eta_{d}^{T}(k)
\end{array}\right]^{T}, \\
& \eta_{1}(k)=\left[\begin{array}{c}
e(k-1) \\
e(k-2) \\
\vdots \\
e\left(k-\rho_{1}+1\right)
\end{array}\right] \\
& \eta_{i}(k)=\left[\begin{array}{c}
e\left(k-\rho_{i-1}\right) \\
e\left(k-\rho_{i-1}-1\right) \\
\vdots \\
e\left(k-\rho_{i}+1\right)
\end{array}\right], \quad i=2,3, \ldots, d .
\end{aligned}
$$

Then, the closed-loop NPC system with constant distributed delays is as follows:

$$
\eta(k+1)=(\Gamma+\Delta \Gamma) \eta(k)
$$

where

$$
\begin{gathered}
\Gamma=\left[\begin{array}{cc}
\Gamma_{11} & B \Gamma_{12} \\
\Gamma_{21} & \Gamma_{22}
\end{array}\right], \\
\Gamma_{11}=\Omega_{11}, \quad \Gamma_{21}=\left[\begin{array}{cc}
0_{n \times n} & I_{n \times n} \\
0_{(d-2) n \times n} & 0_{(d-2) n \times n}
\end{array}\right], \\
\Gamma_{22}=\left[\begin{array}{cc}
0_{n \times(d-2) n} & 0_{n \times n} \\
I_{(d-2) n \times(d-2) n} & 0_{(d-2) n \times n}
\end{array}\right],
\end{gathered}
$$




$$
\begin{gathered}
\Gamma_{12}=\left[\begin{array}{cccc}
\Gamma_{121} & \Gamma_{122} & \cdots & \Gamma_{12 d} \\
0_{n \times n} & 0_{n \times n} & \cdots & 0_{n \times n}
\end{array}\right], \\
\Gamma_{121}=\left[\begin{array}{llll}
K L C & K A L C & \cdots & K A^{\rho_{1}-2} L C
\end{array}\right], \\
\Gamma_{12 i}=\left[\begin{array}{llll}
\Gamma_{12 i 1} & \Gamma_{12 i 2} & \cdots & \Gamma_{12 i\left(\rho_{i}-1\right)}
\end{array}\right] \\
i=2,3, \ldots, d, \\
\Gamma_{12 i 1}=\sum_{j \in W_{i}} \Pi_{j} K A^{\rho_{i-1}-1} L C, \\
\Gamma_{12 i 2}=\sum_{j \in W_{i}} \Pi_{j} K A^{\rho_{i-1}} L C, \\
\vdots \\
\Gamma_{12 i\left(\rho_{i}-1\right)}=\sum_{j \in W_{i}} \Pi_{j} K A^{\rho_{i}-2} L C, \\
\Delta \Gamma=\left[\begin{array}{c}
\Delta \Gamma_{11} \\
0_{(d-1) n \times 2 n}
\end{array} 0_{(d-1) n \times(d-1) n} \Delta B \Gamma_{12}\right. \\
\Delta \Gamma_{11}=\Delta \Omega_{11} .
\end{gathered}
$$

It is clear that system (40) is a standard uncertain system and the stability result is as follows.

Theorem 3. For the NPC system (40) with constant distributed delays, given $\rho_{i}, i \in D$, and controller $K$ and observer $L$, if and only if there exists a matrix $Y$ and a scalar $\gamma>0$ such that the following matrix inequality

$$
\left[\begin{array}{ccc}
-Y & Y \Gamma^{T} & Y H^{T} \\
\Gamma Y & -Y+J J^{T} & 0 \\
H Y & 0 & -\gamma I
\end{array}\right]<0
$$

holds, then the closed-loop NPC system (40) is stable, where

$$
\begin{aligned}
J & =\left[\begin{array}{c}
E \\
E \\
0_{\left(n_{f}-1\right) \times n}
\end{array}\right], \\
H & =\left[\begin{array}{lll}
G_{1}-G_{2} K & G_{2} K & G_{2} \Gamma_{12}
\end{array}\right] .
\end{aligned}
$$

Proof. It can be obtained by (2) that $\Delta \Gamma$ has the structure $\Delta \Gamma=$ $J F(k) H$. Following the robust control system results such as [33], it can be obtained that system (40) is stable if and only if there exists a matrix $P$ such that the following inequality holds:

$$
(\Gamma+\Delta \Gamma)^{T} P(\Gamma+\Delta \Gamma)-P<0 .
$$

Then, similar to the procedure in Theorem 2, the stability result can be obtained readily. The rest of the proof is thus omitted.

Assume that there are no model uncertainties; that is, $\Delta A=0$ and $\Delta B=0$, and then closed-loop NPC system (40) with constant distributed delays can be represented in a more concise form as follows:

$$
\eta(k+1)=\Lambda \eta(k),
$$

where

$$
\begin{aligned}
\Lambda & =\left[\begin{array}{cc}
A-B K & \Lambda_{12} \\
0 & \Lambda_{22}
\end{array}\right], \\
\Lambda_{12} & =B \Gamma_{12}, \\
\Lambda_{22} & =\operatorname{diag}\{A-L C, A-L C, \ldots, A-L C\} .
\end{aligned}
$$

Clearly, a necessary and sufficient condition for the stability of the system (45) is the eigenvalues of $A-B K$ and $A-L C$ that are within the unit circle, which means that the stability of the closed-loop NPC system (45) is not related to the distributed delays. This extends the results in [20] to the multiple forward channels case.

\section{Illustrative Example}

To illustrate the effectiveness of the modified NPC with multiple forward channels, we consider the two-axis example of a three-axis milling machine. More details about the example can be referred to [34]. The parameters of the nominal model and the uncertain parts for the system are chosen as follows:

$$
\begin{aligned}
& A=\left[\begin{array}{cccc}
1.0000 & 0.0461 & 0 & 0 \\
0 & 0.1624 & 0 & 0 \\
0 & 0 & 1.0000 & 0.0466 \\
0 & 0 & 0 & 0.1676
\end{array}\right] \\
& B=\left[\begin{array}{cc}
1.5287 & 0 \\
23.7463 & 0 \\
0 & 1.5458 \\
0 & 24.0982
\end{array}\right], \quad C=\left[\begin{array}{llll}
1 & 0 & 0 & 0 \\
0 & 0 & 1 & 0
\end{array}\right] \text {, } \\
& E=\left[\begin{array}{cc}
h_{1} & 0 \\
h_{2} & 0 \\
0 & h_{3} \\
0 & h_{4}
\end{array}\right], \quad F(k)=\left[\begin{array}{cc}
\sin k & 0 \\
0 & \sin k
\end{array}\right], \\
& G_{1}=\left[\begin{array}{cccc}
0 & g_{1} & 0 & 0 \\
0 & 0 & 0 & g_{2}
\end{array}\right], \quad G_{2}=\left[\begin{array}{cc}
g_{3} & 0 \\
0 & g_{4}
\end{array}\right],
\end{aligned}
$$

where $h_{1}=h_{3}=0.05, h_{2}=h_{4}=0.3, g_{1}=g_{2}=1$, and $g_{3}=g_{4}=40$. The sampling period is chosen as $0.1 \mathrm{~s}$. Clearly, $F^{T}(k) F(k) \leq I$. The observer gain matrix $L$ and state feedback control gain matrix $K$ are calculated by pole placement method and Lyapunov method, respectively. They are given as follows:

$$
\begin{aligned}
L & =\left[\begin{array}{cccc}
0.4715 & 5.1526 & 0.1413 & -0.4592 \\
-0.0093 & -1.3443 & 0.4585 & 5.0377
\end{array}\right]^{T}, \\
K & =\left[\begin{array}{cccc}
0.0401 & 0.0083 & 0 & 0 \\
0 & 0 & 0.0395 & 0.0084
\end{array}\right] .
\end{aligned}
$$




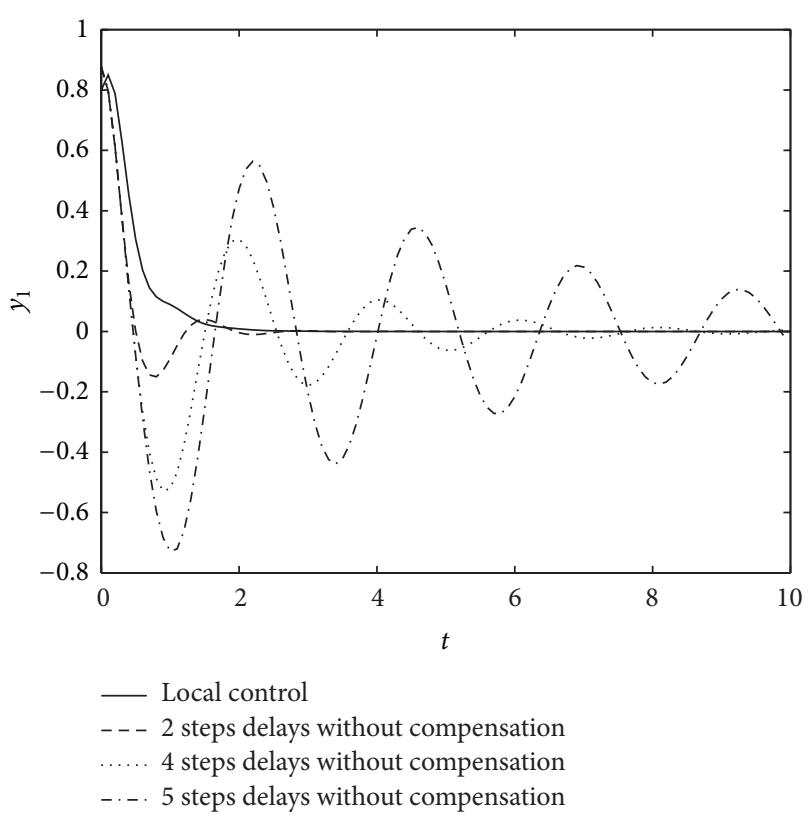

FIgURE 2: Trajectory of $y_{1}$ without NPC compensation.

In the simulation, the system model for the plant and the initial condition are chosen to be

$$
\begin{aligned}
A+\Delta A & =\left[\begin{array}{cccc}
1.0000 & 0.0627 & 0 & 0 \\
0 & 0.3613 & 0 & 0 \\
0 & 0 & 1.0000 & 0.0636 \\
0 & 0 & 0 & 0.3731
\end{array}\right], \\
B+\Delta B & =\left[\begin{array}{cc}
2.1060 & 0 \\
33.0988 & 0 \\
0 & 2.1313 \\
0 & 36.6922
\end{array}\right], \\
x(0) & =\left[\begin{array}{lll}
0.8 & 0.8 & 1
\end{array}\right] .
\end{aligned}
$$

We can see from the structure of the system matrices that $y_{1}$ and $y_{2}$ are independent of each other. It is assumed that there are 2 forward channels for the system and that $y_{1}$ and $y_{2}$ are transmitted via channels 1 and 2 , respectively.

First, the effects of NPC for uncertain NCSs with delays in the forward channels are considered. Let the delays in both channel 1 and channel 2 be equal and constant and see the system performance. Take $y_{1}$ for example, as shown in Figure 2; the system performance becomes worse with larger delay and the system is unstable with more than 6 steps delays. The trajectory of $y_{1}$ with NPC is shown in Figure 3, from which we can see that the delays are effectively compensated. By applying Theorem 3, the closed-loop NPC system is still stable with 10 steps delay.

Then, we consider the effectiveness of the modified NPC with distributed delays and packet dropouts. In this simulation of constant case, the delays in channel 1 and channel 2 are chosen to be 4 steps and 3 steps, respectively. By Theorem 3 it can be obtained that the corresponding closedloop system is stable. From the analysis, it can be inferred that the trajectory of $y_{1}$ will be the same by using modified and

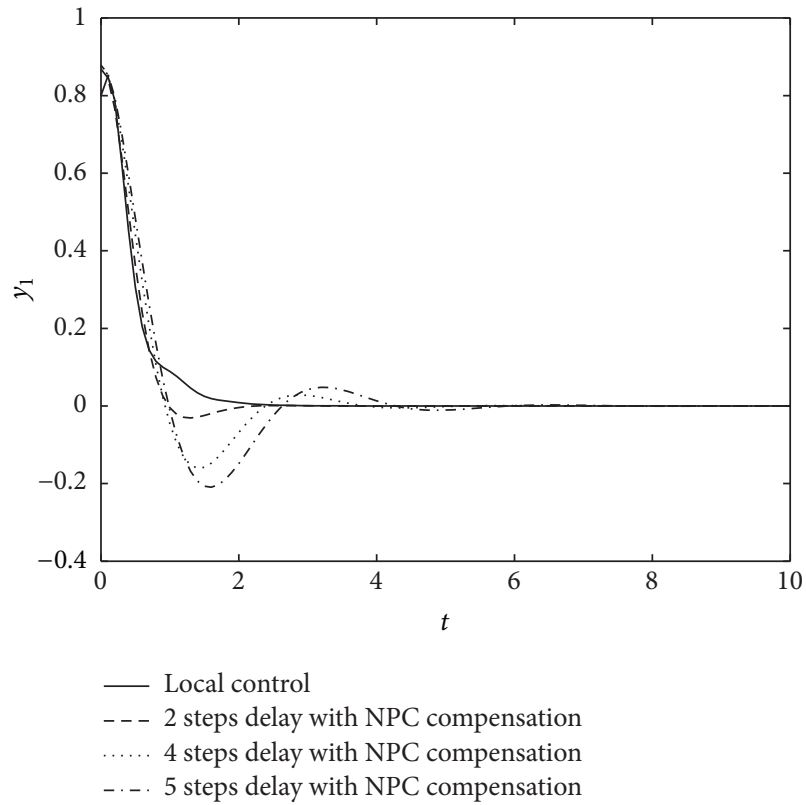

FIgURE 3: Trajectory of $y_{1}$ with NPC compensation.

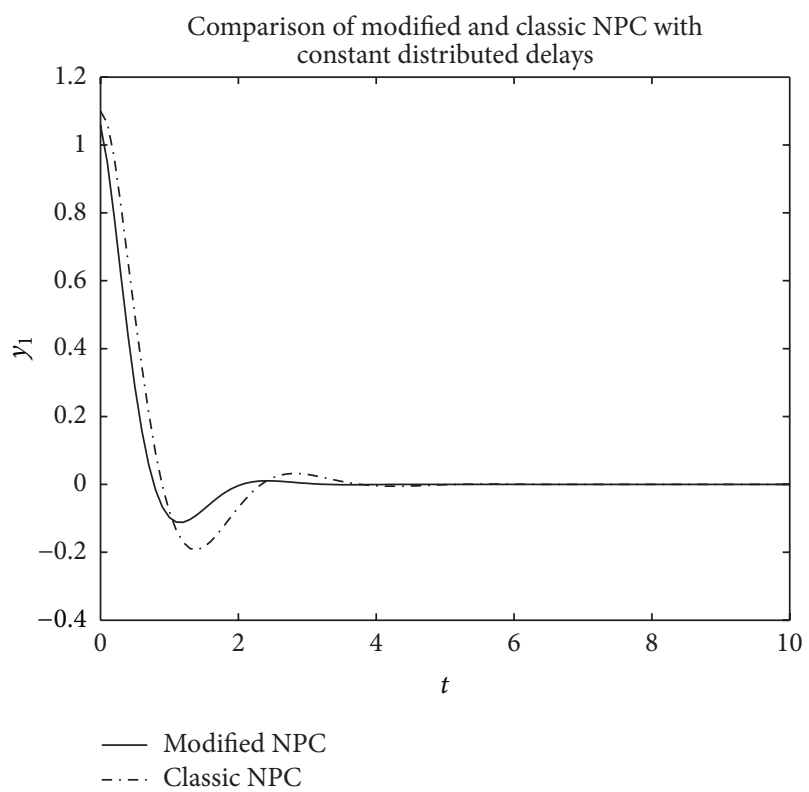

FIGURE 4: Comparison of modified and classic NPC with distributed constant delays.

classic NPC since the delay in channel 1 is always the largest. While the performance of $y_{2}$ will be better by using modified NPC than classic one. Figure 4 show the trajectory of $y_{2}$ with modified and classic NPC, supporting the theory.

Finally, the case of random distributed delays and packet dropouts is simulated. $n_{d}$ and $n_{p}$ are chosen to be 4 and 2, respectively. That is to say, the delays in channels 1 and 2 are both random between 1 step and 4 steps, and the number of consecutive packet dropouts in both channels is up to 2 steps. By Theorem 2, it follows that the closed-loop system is 

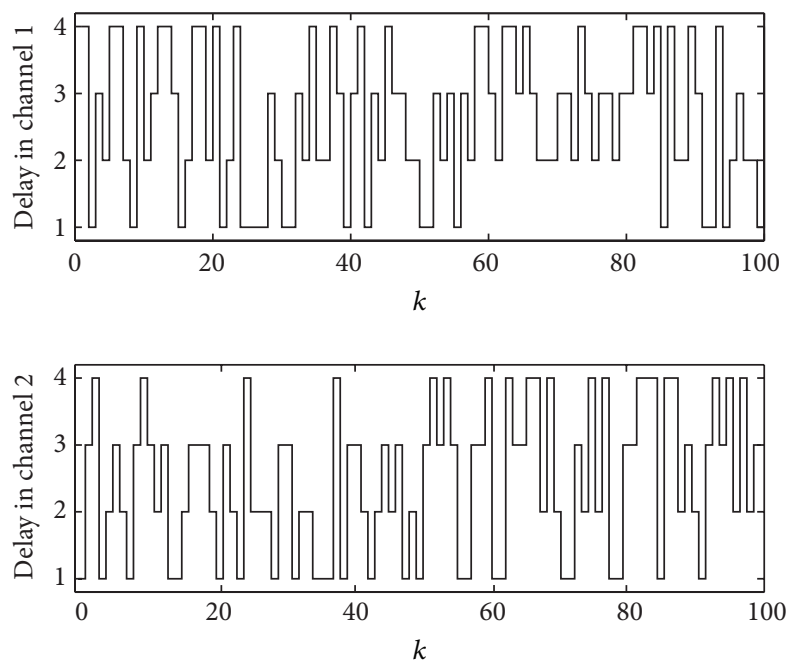

FIgURE 5: Random distributed delays.
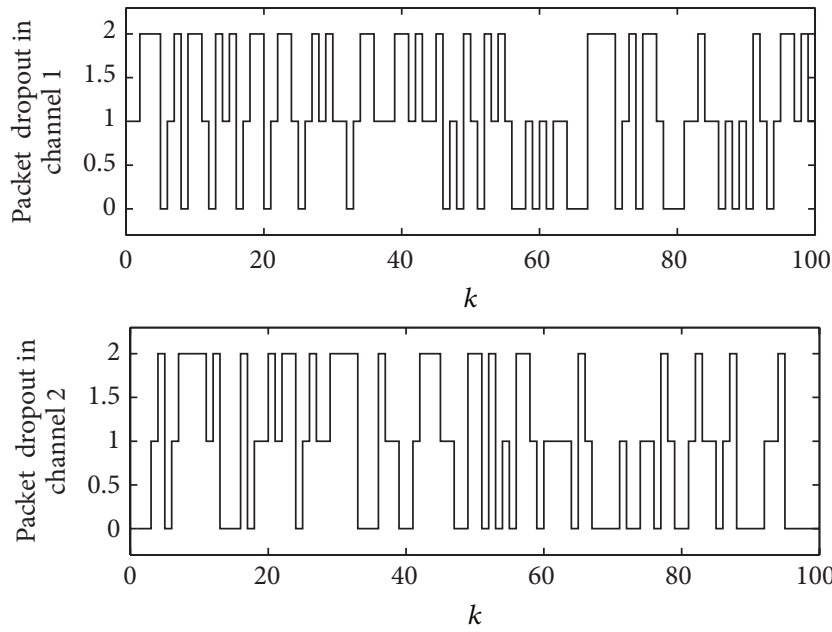

FIGURE 6: Random distributed packet dropouts process.

stable. The simulated distributed random delays and packet dropouts process are shown in Figures 5 and 6, respectively. The corresponding trajectories of $y_{1}$ and $y_{2}$ are shown in Figures 7 and 8 , respectively. Clearly, we can see that the modified NPC achieves better performance than classic NPC method for this example.

\section{Conclusions}

This paper studied the design and stability analysis of uncertain networked predictive control systems with distributed delays and packet dropouts in the forward channels. A modified NPC was proposed, in which the key point is an improved control signal selection scheme. The CSS with distributed prediction length uses the most recent data and hence can make modified NPC achieve better control performance. Stability analysis results are obtained for both constant and random cases. They are formulated as linear matrix inequalities and can be readily checked. Moreover, it is shown

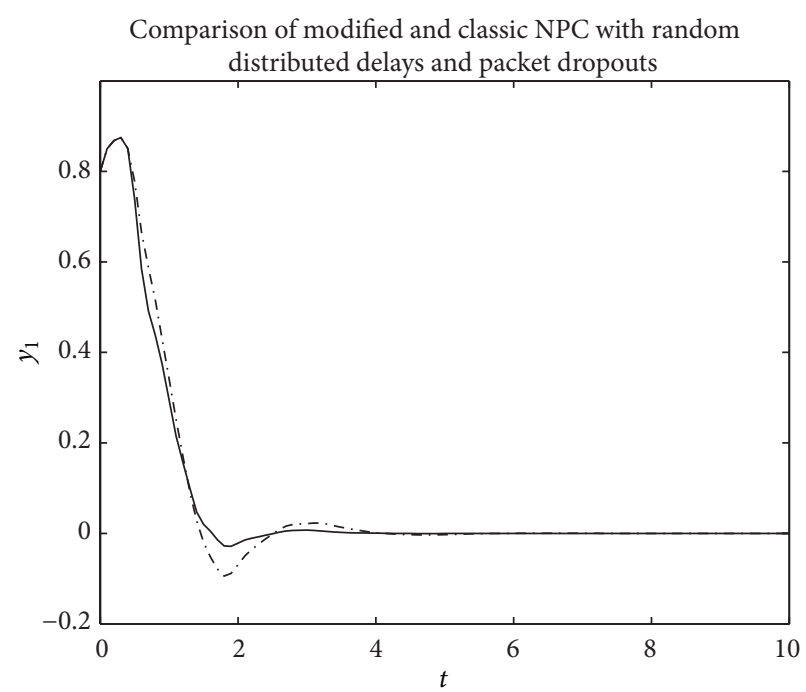

— Modified NPC

-.. Classic NPC

Figure 7: Trajectory of $y_{1}$ with modified and classic NPC with distributed random delays and packet dropouts.

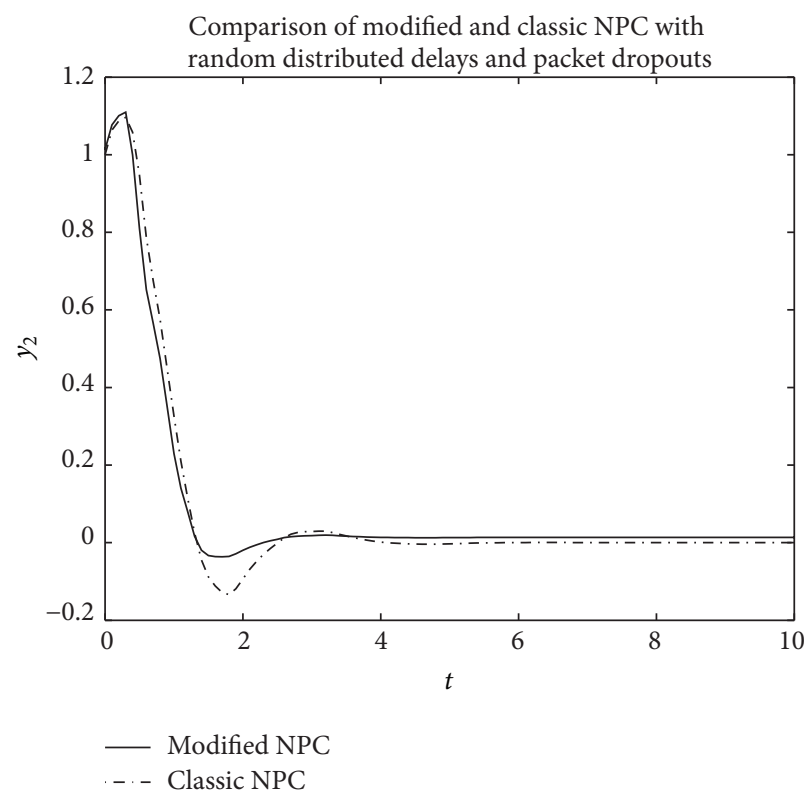

FIGURE 8: Trajectory of $y_{2}$ of modified and classic NPC with distributed random delays and packet dropouts.

that the stability of the closed-loop NPC system is not related to the distributed delays when they are constant and system model is accurate. An example was given to show that the modified NPC method achieves better performance than classic NPC in the case of distributed delays and packet dropouts in the forward channels. 


\section{Conflict of Interests}

The author declares that there is no conflict of interests regarding the publication of this paper.

\section{Acknowledgments}

This research work was supported by the National Nature Science Foundation of China (Grant no. 61304040) and the Nature Science Foundation of Zhejiang Province (Grant no. LQ13F030005).

\section{References}

[1] W. Zhang, M. S. Branicky, and S. M. Philips, "Stability of networked control systems," IEEE Control Systems Magazine, vol. 21, no. 1, pp. 84-99, 2001.

[2] L. X. Zhang, H. J. Gao, and O. Kaynak, "Network-induced constraints in networked control systems-a survey," IEEE Transactions on Industrial Informatics, vol. 9, no. 1, pp. 403-416, 2013.

[3] H. Gao, T. Chen, and J. Lam, "A new delay system approach to network-based control," Automatica, vol. 44, no. 1, pp. 39-52, 2008.

[4] H. Yan, H. Shi, H. Zhang, and F. Yang, "Quantized $H_{\infty}$ control for networked systems with communication constraints," Asian Journal of Control, vol. 15, no. 5, pp. 1468-1476, 2013.

[5] J. P. Hespanha, P. Naghshtabrizi, and Y. Xu, "A survey of recent results in networked control systems," Proceedings of the IEEE, vol. 95, no. 1, pp. 138-172, 2007.

[6] Y.-G. Sun and Q.-Z. Gao, "Stability and stabilization of networked control system with forward and backward random time delays," Mathematical Problems in Engineering, vol. 2012, Article ID 834643, 15 pages, 2012.

[7] H. Zhang, H. Yan, F. Yang, and Q. Chen, "Quantized control design for impulsive fuzzy networked systems," IEEE Transactions on Fuzzy Systems, vol. 19, no. 6, pp. 1153-1162, 2011.

[8] D. Zhang, Q. G. Wang, L. Yu, and Q. K. Shao, " $H_{\infty}$ filtering for networked systems with multiple time-varying transmissions and random packet dropouts," IEEE Transactions on Industrial Informatics, vol. 9, no. 3, pp. 1705-1716, 2013.

[9] B. Chen, L. Yu, and W.-A. Zhang, "Robust Kalman filtering for uncertain state delay systems with random observation delays and missing measurements," IET Control Theory \& Applications, vol. 5, no. 17, pp. 1945-1954, 2011.

[10] H. Zhang, Q. Chen, H. Yan, and J. Liu, "Robust $H_{\infty}$ filtering for switched stochastic system with missing measurements," IEEE Transactions on Signal Processing, vol. 57, no. 9, pp. 3466-3474, 2009.

[11] D. Yue, E. Tian, and Q.-L. Han, "A delay system method for designing event-triggered controllers of networked control systems," IEEE Transactions on Automatic Control, vol. 58, no. 2, pp. 475-481, 2013.

[12] H. Yan, Z. Su, H. Zhang, and F. Yang, "Observer-based $H_{\infty}$ control for discrete-time stochastic systems with quantisation and random communication delays," IET Control Theory \& Applications, vol. 7, no. 3, pp. 372-379, 2013.

[13] L. Zhang, Y. Shi, T. Chen, and B. Huang, "A new method for stabilization of networked control systems with random delays," IEEE Transactions on Automatic Control, vol. 50, no. 8, pp. 11771181, 2005.
[14] M. C. F. Donkers, W. P. M. H. Heemels, N. van de Wouw, and L. Hetel, "Stability analysis of networked control systems using a switched linear systems approach," IEEE Transactions on Automatic Control, vol. 56, no. 9, pp. 2101-2115, 2011.

[15] W.-A. Zhang and L. Yu, "A robust control approach to stabilization of networked control systems with time-varying delays," Automatica, vol. 45, no. 10, pp. 2440-2445, 2009.

[16] M. Yu, L. Wang, T. Chu, and G. Xie, "Stabilization of networked control systems with data packet dropout and network delays via switching system approach," in Proceedings of the 43rd IEEE Conference on Decision and Control (CDC '04), pp. 3539-3544, Paradise Island, Bahamas, December 2004.

[17] P. Seiler and R. Sengupta, "An $H_{\infty}$ approach to networked control," IEEE Transactions on Automatic Control, vol. 50, no. 3, pp. 356-364, 2005.

[18] G. P. Liu, J. X. Mu, D. Rees, and S. C. Chai, "Design and stability analysis of networked control systems with random communication time delay using the modified MPC,' International Journal of Control, vol. 79, no. 4, pp. 288-297, 2006.

[19] G.-P. Liu, Y. Xia, J. Chen, D. Rees, and W. Hu, "Networked predictive control of systems with random network delays in both forward and feedback channels," IEEE Transactions on Industrial Electronics, vol. 54, no. 3, pp. 1282-1297, 2007.

[20] G. P. Liu, "Predictive controller design of networked systems with communication delays and data loss," IEEE Transactions on Circuits and Systems II: Express Briefs, vol. 57, no. 6, pp. 481-485, 2010.

[21] W. Hu, G. Liu, and D. Rees, "Event-driven networked predictive control," IEEE Transactions on Industrial Electronics, vol. 54, no. 3, pp. 1603-1613, 2007.

[22] R. Wang, G.-P. Liu, W. Wang, D. Rees, and Y.-B. Zhao, " $H_{\infty}$ control for networked predictive control systems based on the switched Lyapunov function method," IEEE Transactions on Industrial Electronics, vol. 57, no. 10, pp. 3565-3571, 2010.

[23] J. H. Zhang, Y. Q. Xia, and P. Shi, "Design and stability analysis of networked predictive control systems," IEEE Transactions on Control Systems and Technology, vol. 21, no. 4, pp. 1495-1501, 2013.

[24] Y. Xia, W. Xie, B. Liu, and X. Wang, "Data-driven predictive control for networked control systems," Information Sciences, vol. 235, pp. 45-54, 2013.

[25] G. Pin and T. Parisini, "Networked predictive control of uncertain constrained nonlinear systems: recursive feasibility and input-to-state stability analysis," IEEE Transactions on Automatic Control, vol. 56, no. 1, pp. 72-87, 2011.

[26] A. Onat, T. Naskali, E. Parlakay, and O. Mutluer, "Control over imperfect networks: model-based predictive networked control systems," IEEE Transactions on Industrial Electronics, vol. 58, no. 3, pp. 905-913, 2011.

[27] Y.-B. Zhao, J. Kim, and G.-P. Liu, "Error bounded sensing for packet-based networked control systems," IEEE Transactions on Industrial Electronics, vol. 58, no. 5, pp. 1980-1989, 2011.

[28] Y.-B. Zhao, J. Kim, X.-M. Sun, and G.-P. Liu, "On the delay effects of different channels in Internet-based networked control systems," International Journal of Systems Science, vol. 44, no. 11, pp. 2119-2129, 2013.

[29] H. B. Song, G. P. Liu, and L. Yu, "Networked predictive control of uncertain systems with multiple feedback channels," IEEE Transactions on Industrial Electronics, vol. 60, no. 11, pp. 52285238, 2013. 
[30] G.-P. Liu, "Analysis of networked predictive control systems with uncertainties," in Proceedings of the IEEE International Conference on Systems, Man and Cybernetics (SMC '09), pp. 4819-4824, San Antonio, Tex, USA, October 2009.

[31] Y. B. Zhao, J. Kim, G. H. Yang, and G. P. Liu, "Model-based compensation for multi-packet transmission in networked control systems," in Proceedings of the 50th IEEE Conference on Decision and Control and European Control Conference, pp. 3136-3141, Orlando, Fla, USA, December 2011.

[32] R. Luck and A. Ray, "An observer-based compensator for distributed delays," Automatica, vol. 26, no. 5, pp. 903-908, 1990.

[33] S. Boyd, L. El Ghaoui, E. Feron, and V. Balakrishnan, Linear Matrix Inequalities in System and Control Theory, vol. 15 of SIAM Studies in Applied Mathematics, SIAM, Philadelphia, Pa, USA, 1994.

[34] F.-L. Lian, J. Moyne, and D. Tilbury, "Modelling and optimal controller design of networked control systems with multiple delays," International Journal of Control, vol. 76, no. 6, pp. 591606, 2003. 


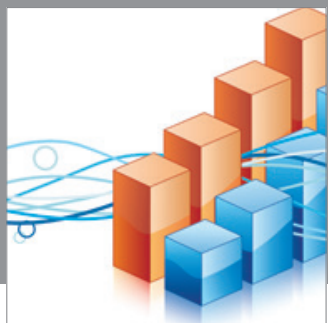

Advances in

Operations Research

mansans

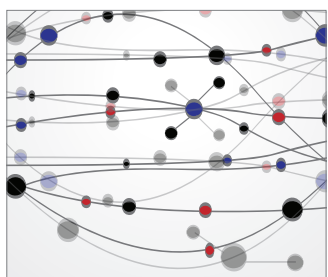

The Scientific World Journal
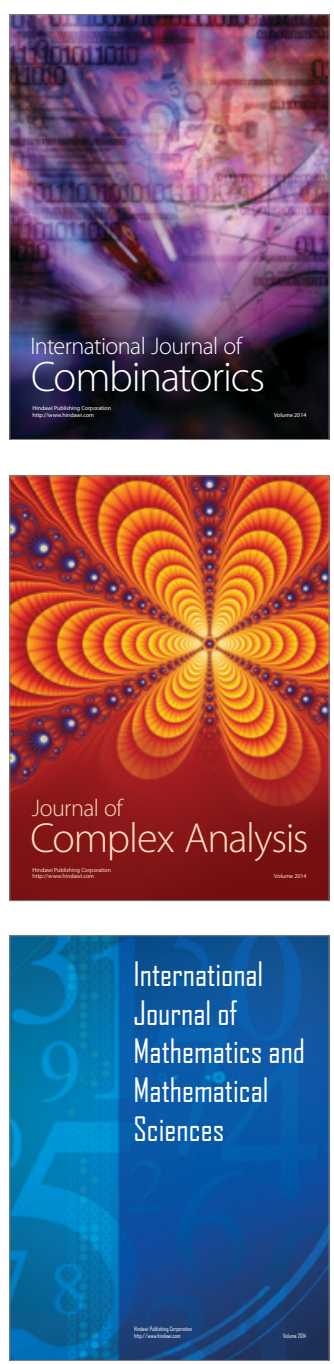
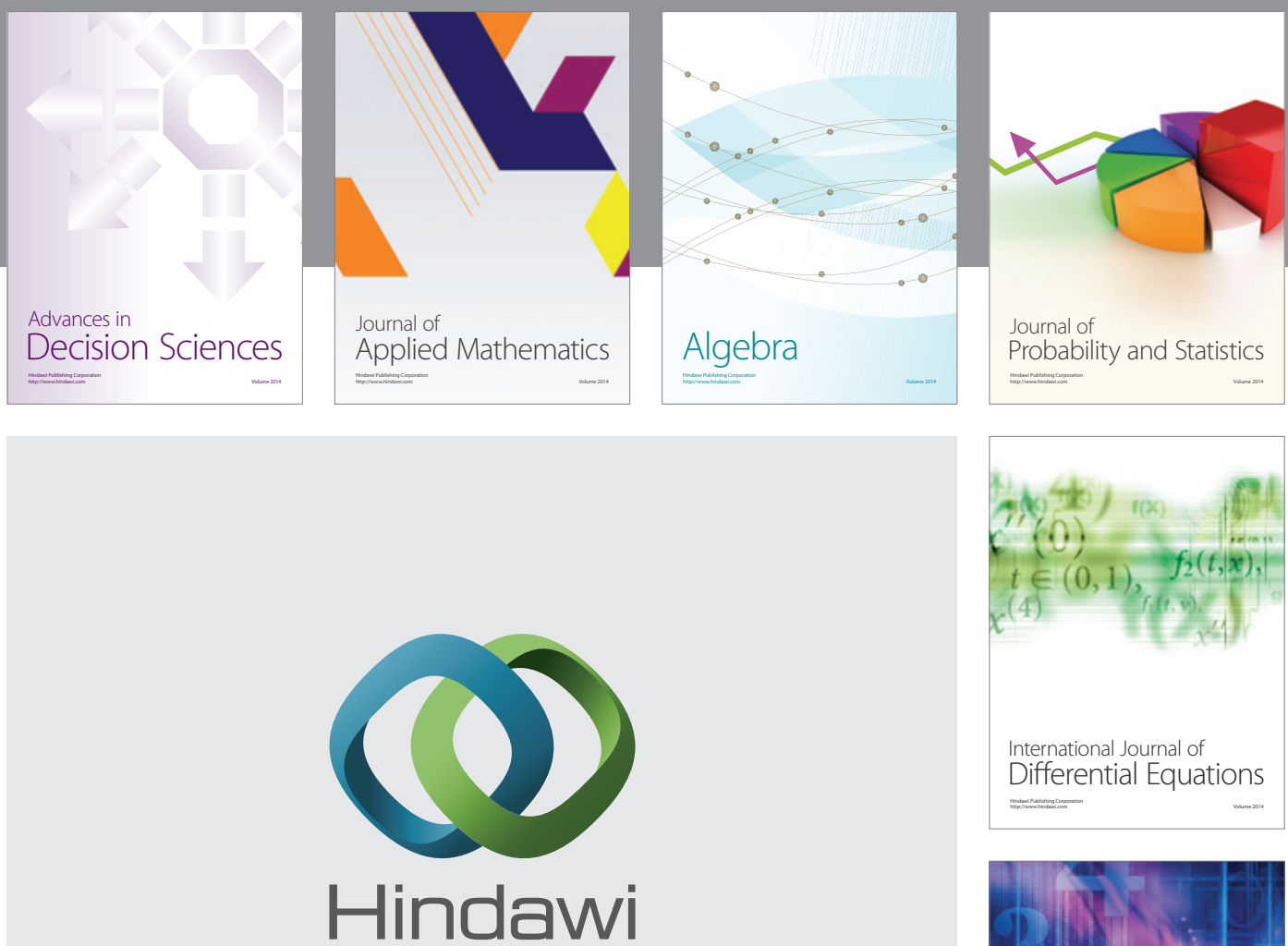

Submit your manuscripts at http://www.hindawi.com
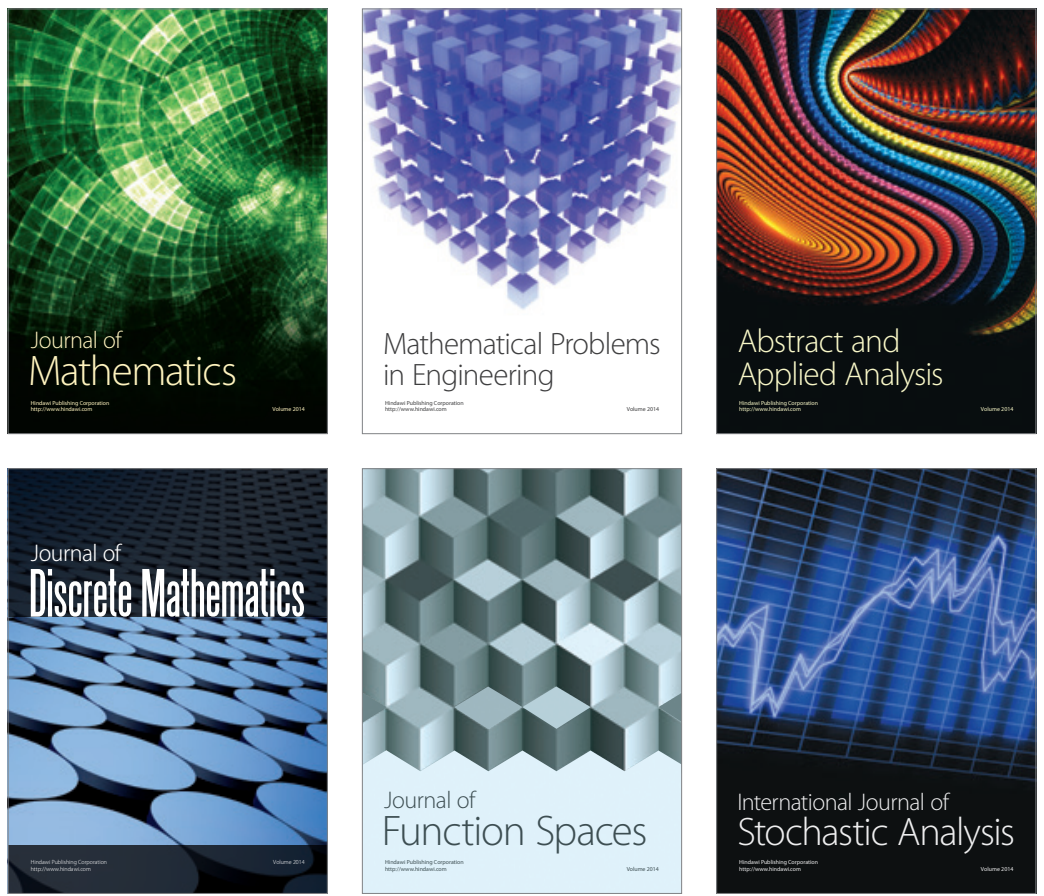

Journal of

Function Spaces

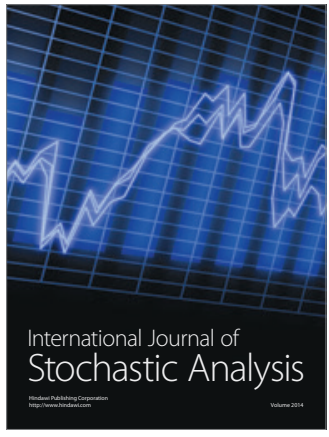

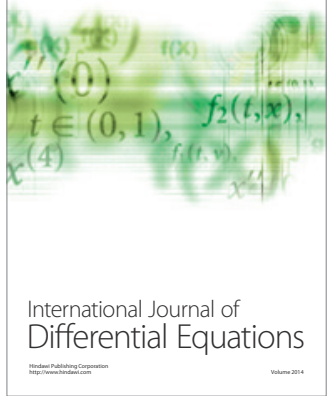
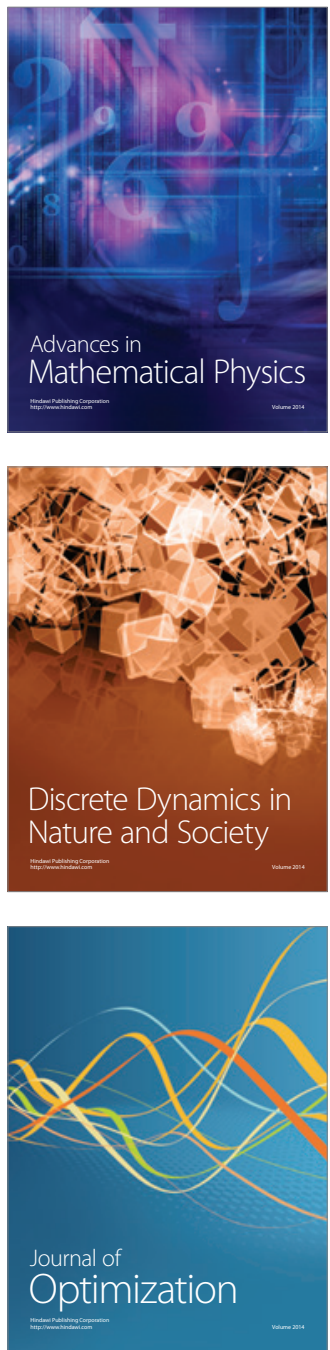